\title{
Temperature-Dependent Defect Dynamics in the Network Glass $\mathrm{SiO}_{2}$
}

\author{
Katharina Vollmayr-Lee ${ }^{1,2, *}$ and Annette Zippelius ${ }^{2,3}$ \\ ${ }^{1}$ Department of Physics and Astronomy, Bucknell University, Lewisburg, Pennsylvania 17837, USA \\ ${ }^{2}$ Georg-August-Universität Göttingen, Institut für Theoretische Physik, \\ Friedrich-Hund-Platz 1, 37077 Göttingen, Germany \\ ${ }^{3}$ Max-Planck-Institut für Dynamik und Selbstorganisation, Bunsenstr. 10, 37073 Göttingen, Germany
}

(Dated: July 8, 2013)

\begin{abstract}
We investigate the long time dynamics of a strong glass former, $\mathrm{SiO}_{2}$, below the glass transition temperature by averaging single particle trajectories over time windows which comprise roughly 100 particle oscillations. The structure on this coarse-grained time scale is very well defined in terms of coordination numbers, allowing us to identify ill-coordinated atoms, called defects in the following. The most numerous defects are $\mathrm{OO}$ neighbors, whose lifetimes are comparable to the equilibration time at low temperature. On the other hand $\mathrm{SiO}$ and OSi defects are very rare and short lived. The lifetime of defects is found to be strongly temperature dependent, consistent with activated processes. Single-particle jumps give rise to local structural rearrangements. We show that in $\mathrm{SiO}_{2}$ these structural rearrangements are coupled to the creation or annihilation of defects, giving rise to very strong correlations of jumping atoms and defects.
\end{abstract}

PACS numbers: 61.20.Lc, 61.20.Ja, 64.70.ph, 61.43.Fs

\section{INTRODUCTION}

Amorphous $\mathrm{SiO}_{2}$, or silica, has many fascinating features. Silica is of importance in geology, chemistry, physics and industrial applications. To classify the huge variety of glass formers in general [1-3], we distinguish fragile and strong glass formers [3 $[5]$. Silica is a typical strong glass former, i.e. the shear viscosity exhibits Arrhenius behavior at low temperature and pressure. With increasing temperature $\mathrm{SiO}_{2}$ undergoes at $T_{\mathrm{c}}$ a strong to fragile transition [6] and for large pressure critical behavior of a liquid-liquid transition has been observed [7].

We investigate here $\mathrm{SiO}_{2}$ via molecular dynamics simulations using the van Beest-Kramer-van Santen (BKS) potential [8] for the particle interactions. Since previous simulations had shown that the BKS-potential is a very good model for real silica $([6,9-11]$ and references therein) many simulations with the BKS-potential followed, giving us insight into the phase diagram [12 15], energy landscape [16 20], specific heat 21], vibrational spectrum 11, 22 25], dynamic heterogeneities [26 29], and aging [30 32] [33].

For temperatures below $T_{\mathrm{c}}=3330 \mathrm{~K}[\underline{6}], \mathrm{BKS}_{-} \mathrm{SiO}_{2}$ is a strong glass former. A striking similarity with fragile glass formers has been found for single particle jump dynamics [32]. This is surprising at first sight, because the local structures in fragile and strong glasses differ considerably. In fragile glasses the concept of a cage is well established and jumps are interpreted as particles escaping from their cage. The underlying structure in $\mathrm{SiO}_{2}$, on the other hand, is based on randomly connected tetrahedra, forming a macroscopic random network. Even though the macroscopic network is random, coordination

\footnotetext{
*kvollmay@bucknell.edu
}

numbers are very well defined, so that defects are easily identified. In this paper we address the question to what extent jump events are correlated with the creation of defects.

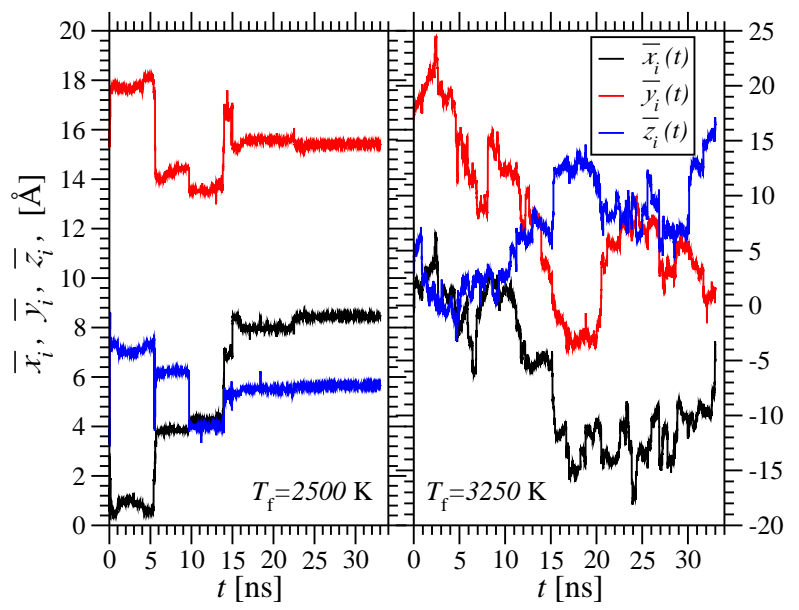

FIG. 1. (color online) Examples for time-averaged single particle (O-atom) trajectories for $T_{\mathrm{i}}=5000 \mathrm{~K}$ at $T_{\mathrm{f}}=2500 \mathrm{~K}$ (left figure) and at $T_{\mathrm{f}}=3250 \mathrm{~K}$ (right figure).

Our focus is on structural rearrangements well inside the glassy phase. At low temperature, we expect a clear separation of time scales, such that oscillations around preferred positions are characterized by short time scales, whereas (rare) structural changes occur on much longer time scales. To illuminate the latter, we filter out short time oscillations by averaging particle trajectories and analyze the time-averaged single particle $i$ trajectories $\overline{\mathbf{r}_{i}}(t)$ in terms of jumps of particles and creation and annihilation of defects. We find a clear temperature dependence of the time-averaged dynamics which is already apparent in single particle trajectories; an example is shown 
in Fig. 1. Whereas for the lowest temperatures under consideration jump events are well separated in time by long quiescent periods, this separation of time scales is gradually lost, when the glass transition is approached from below.

Our approach is similar in spirit to the analysis of inherent structures [16 19, 34, 35], where instantaneous configurations are quenched to their local potential energy minimum. In [16, 18] energy minimized configurations have been analyzed for $\mathrm{BKS}_{-} \mathrm{SiO}_{2}$ in order to explain the observed crossover from strong to fragile behavior. We expect the time-averaged trajectories to be strongly correlated with the corresponding inherent structures. However, in contrast to inherent structures, our approach allows us to study the dynamics of local structural rearrangements. Time averaged trajectories have been studied previously in refs. [36, 37] for soft sphere glasses and in [38] for Lennard-Jones glasses. Keys et al. 38] use time persistent particle displacements to identify those excitations which are responsible for structural relaxation.

After introducing the model in section II] we show in section III that the radial distribution functions of the time-averaged trajectories are considerably sharpened as compared to the corresponding distributions for the unaveraged trajectories. This implies a stable, well-defined structure on time scales large compared to a typical oscillation period. This time-averaged structure is only weakly temperature dependent. The dynamics on intermediate and long time scales is dominated by defects in the random network structure which are well defined in terms of the coordination number due to the sharp peak structure of the radial distribution function. Number and lifetime of the defects are strongly temperature dependent as discussed in section [V] In section $\nabla$ we show that the jump events as defined in [32] are strongly correlated with the defects in the random network. We summarize our results and draw conclusions in VI.

\section{MODEL AND SIMULATION DETAILS}

To model amorphous $\mathrm{SiO}_{2}$ we used the BKS potential [8]. We carried out molecular dynamics (MD) simulations with $N_{\mathrm{Si}}=112$ silica atoms and $N_{\mathrm{O}}=224$ oxygen atoms and at constant volume $V=(16.920468 \AA)^{3}$, which corresponds to a density of $\rho=2.323 \mathrm{~g} / \mathrm{cm}^{3}$.

At $6000 \mathrm{~K}$ we generated 20 independent configurations, which then were fully equilibrated at initial temperature $T_{\mathrm{i}} \in\{5000 \mathrm{~K}, 3760 \mathrm{~K}\}$ followed by an instantaneous quench to lower temperatures $T_{\mathrm{f}} \in\{2500 \mathrm{~K}, 2750 \mathrm{~K}$, $3000 \mathrm{~K}, 3250 \mathrm{~K}\}$, i.e. to temperatures below $T_{c}=3330 \mathrm{~K}$. Unique to our simulations is that we applied the NoséHoover temperature bath at $T_{\mathrm{f}}$ only for the first $0.327 \mathrm{~ns}$ (NVT) and then continued with constant energy (NVE) for $32.7 \mathrm{~ns}$ to disturb the dynamics minimally. As shown in 31] we confirmed that $T_{\mathrm{f}}$ stayed constant. The MD time step was $1.02 \mathrm{fs}$ and $1.6 \mathrm{fs}$ during the (NVT) and
(NVE) run respectively. For further details of the simulations we refer the reader to 31.

We analyzed the combined (NVT) and (NVE) simulation runs at $T_{\mathrm{f}}$. Specifically, for this paper we focus on major structural events by analyzing time-averaged single particle trajectories $\overline{\mathbf{r}}_{i}\left(t_{l}\right)$ at times $t_{l}=l \Delta t_{\mathrm{av}}$. The typical time scale of an oscillation is around $3 \times 10^{-14} \mathrm{~s}$, roughly twenty times the MD step. The time average is taken over $\Delta t_{\mathrm{av}}$ which has to be chosen large as compared to the oscillation time and sufficiently small to resolve structural rearrangements such as single particle jumps and the creation and annihilation of defects. For most of the data presented below, we have used $\Delta t_{\mathrm{av}}=3.2710^{-12}$ $\mathrm{s}$, allowing for $l=1, \ldots, 10100$ points of the trajectory, but we have checked other values of $\Delta t_{\mathrm{av}}$ as well (see below).

\section{RADIAL DISTRIBUTION FUNCTION AND COORDINATION NUMBER}

We first discuss the structural properties of our system on time scales long compared to a typical oscillation period. To that end we first compute the radial distribution functions for the time-averaged trajectories and compare them to the corresponding quantities for the unaveraged trajectories, representing the structure on microscopic time scales. We then go on to discuss the temperature dependence of the time-averaged structure and the distribution of coordination numbers.

\section{A. Radial distribution function of time-averaged trajectories}

To analyze the local structure implied by the timeaveraged trajectories we compute

$$
g_{\alpha \beta}(r)=\left\langle\frac{V}{N_{\alpha} N_{\beta}} \sum_{i=1}^{N_{\alpha}} \sum_{\substack{j=1 \\ j \neq i}}^{N_{\beta}} \delta\left(|\mathbf{r}|-\left|\overline{\mathbf{r}}_{i j}\left(t_{l}\right)\right|\right\rangle,\right.
$$

where $\alpha, \beta \in\{\mathrm{Si}, \mathrm{O}\}$ (for the case of $\alpha=\beta$ the denominator is $\left.N_{\alpha}\left(N_{\alpha}-1\right)\right), \overline{\mathbf{r}}_{i j}\left(t_{l}\right)$ is defined via the timeaveraged trajectories $\overline{\mathbf{r}}_{i j}\left(t_{l}\right)=\overline{\mathbf{r}}_{i}\left(t_{l}\right)-\overline{\mathbf{r}}_{j}\left(t_{l}\right)$. To increase statistics in all following (unless otherwise specified) the ensemble average $\langle\ldots\rangle$ is obtained via an average over 20 independent simulation runs and an average over 1000 consecutive times, $t_{l}$, starting at a waiting time $t_{\mathrm{w}}=16.35 \mathrm{~ns}$. For all following figures we used $T_{\mathrm{i}}=5000 \mathrm{~K}$. (This choice of $t_{\mathrm{w}}$ results from previous work [32]; we have checked other waiting times as well as different $T_{i}$, see IVB . As will be shown below, the typical relaxation times in our system are larger than $\Delta t_{\mathrm{av}}$, so that configurations at different $t_{l}$ are not completely uncorrelated. Therefore we determine error bars via the 20 independent simulation runs. 


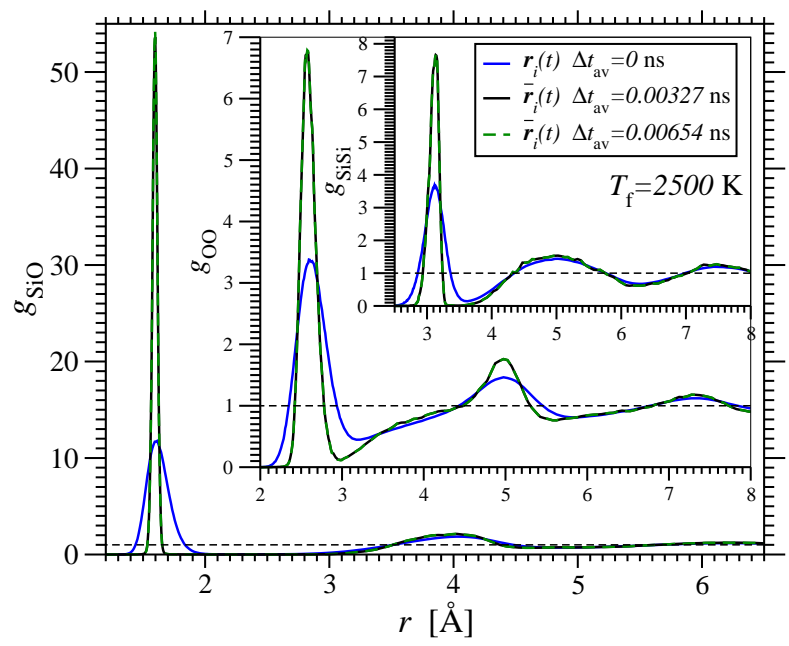

FIG. 2. Radial distribution function $g_{\alpha \beta}(r)$ as defined in Eq. (1) using different time averages $\Delta t_{\mathrm{av}}$ for the time average of $\overline{\mathbf{r}}_{i}(t)$. Here for final temperatures $T_{\mathrm{f}}=2500 \mathrm{~K}$ quenched from $T_{\mathrm{i}}=$ $5000 \mathrm{~K}$.

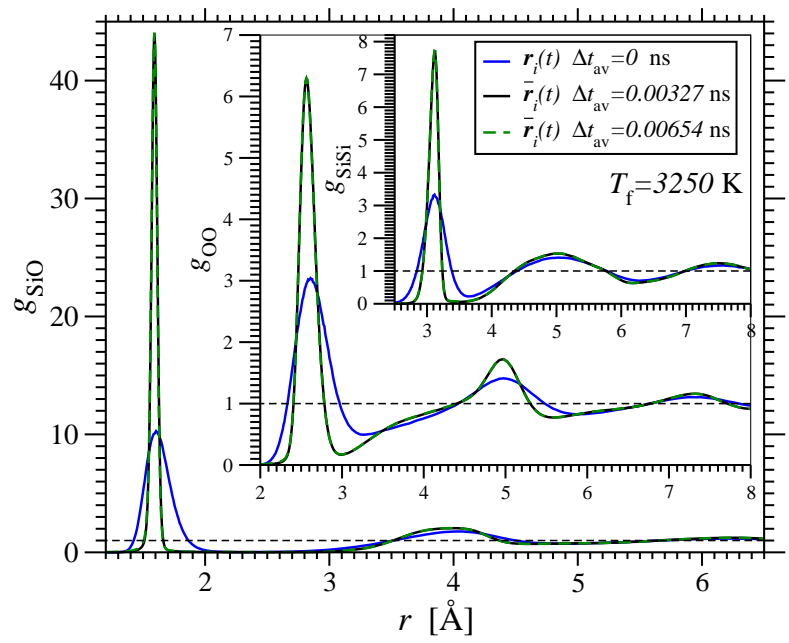

FIG. 3. Radial distribution function as in Fig. 2 but here for final temperature $T_{\mathrm{f}}=3250 \mathrm{~K}$.

In Figs. 2 \& 3 we compare the pair correlation for trajectories with and without time averaging. We conclude that time averaging sharpens the pair correlation drastically, both for low $\left(T_{\mathrm{f}}=2500 \mathrm{~K}\right)$ and high temperatures $\left(T_{\mathrm{f}}=3250 \mathrm{~K}\right)$. The enhancement is particularly strong for the nearest-neighbor peak of $g_{\mathrm{SiO}}$, implying that the structural unit of one tetrahedron with an Si-atom at the center and four O-atoms at the corners is well defined. We obtain the same $g_{\alpha \beta}(r)$ for $\Delta t_{\mathrm{av}}=0.00327 \mathrm{~ns}$ and $\Delta t_{\mathrm{av}}=0.00654 \mathrm{~ns}$, supporting the separation of time scales, so that time averaging over $\Delta t_{\mathrm{av}}$ allows us to filter out the main structural features of an underlying network that is highly ordered in the sense that nearest neighbor distances are well-defined on time scales of the order of 0.005 ns.

\section{B. Temperature dependence of the time-averaged structure}

Next we investigate, how the time-averaged structure depends on temperature. In Figs. 4 and 5 we compare the radial distribution function, as defined in Eq. (1), for four different temperatures. For $g_{\mathrm{SiO}}(r)$ (see Fig. (4) we find an increase of roughly $20 \%$ for the temperature range investigated. Compared to the increase by a factor of $\sim 5$ due to time averaging this is a rather mild effect. Similarly, for $g_{\mathrm{OO}}(r)$ (see Fig. 5) the first peak is enhanced by roughly $5 \%$, again small as compared to the increase by a factor of $\sim 2$ due to time averaging. For distances beyond nearest neighbors, the radial distribution function of the time-averaged configurations is basically temperature independent. We conclude that the time-averaged structure is only weakly temperature dependent.

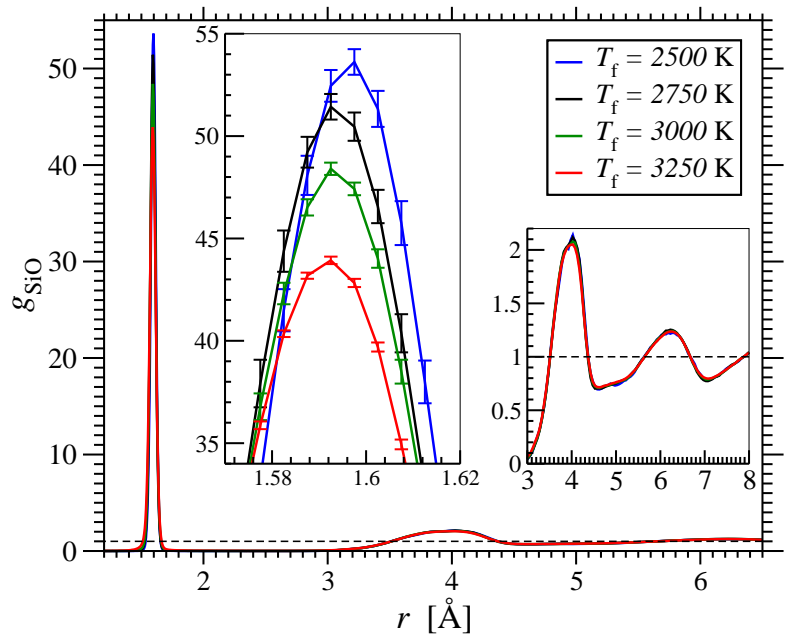

FIG. 4. (color online) Radial distribution function $g_{\mathrm{SiO}}(r)$ for final temperatures $T_{\mathrm{f}}$. The left inset is an enlargement of the first peak and the right inset is an enlargement of farther peaks.

\section{Coordination numbers}

For all investigated time-averaged $g_{\alpha \beta}(r)$ the first peak is very sharp and the minimum between the first and second peaks is very deep, indicating a well-defined first neighbor shell. We therefore define for each particle $i$ of particle type $\alpha$ at time $t_{l}$ the coordination number $z_{i}^{\alpha \beta}\left(t_{l}\right)$ to be the number of other particles $j$ of type $\beta$ which satisfy

$$
\left|\overline{\mathbf{r}}_{i}\left(t_{l}\right)-\overline{\mathbf{r}}_{j}\left(t_{l}\right)\right|<r_{\min }^{\alpha \beta}
$$

where $r_{\min }^{\mathrm{SiSi}}=3.42 \AA, r_{\mathrm{min}}^{\mathrm{SiO}}=2.40 \AA$ and $r_{\min }^{\mathrm{OO}}=3.00 \AA$. The resulting coordination number distributions $P_{\alpha \beta}$ are plotted in Figs. 66 \& 7, ensemble averaged as in Eq. (1).

Please note that the distributions $P(z)$ are so sharply peaked that we chose a logarithmic scale. At $T_{\mathrm{f}}=2500 \mathrm{~K}$ 


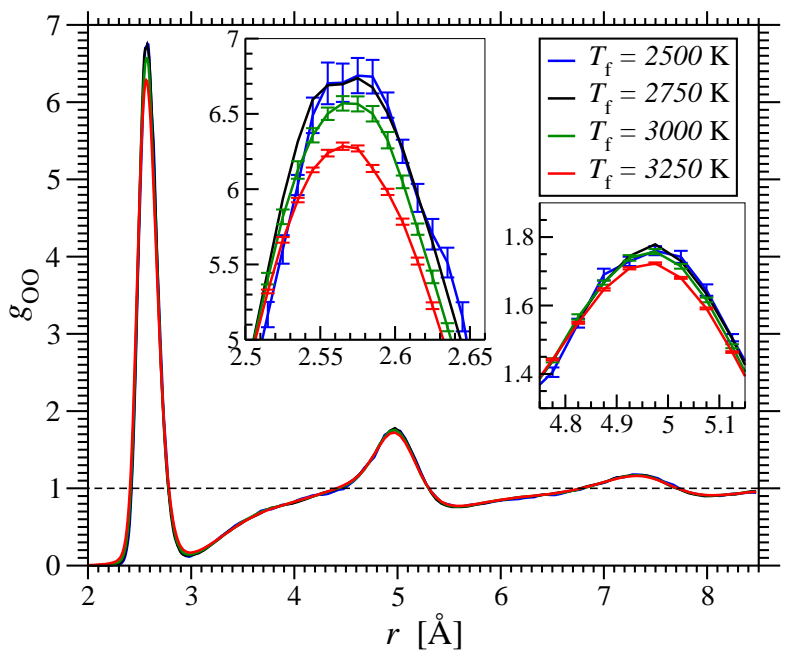

FIG. 5. (color online) Similar to Fig. 4 the radial distribution function, but here for $g_{\mathrm{OO}}(r)$. The insets are enlargements of the first and second peak.

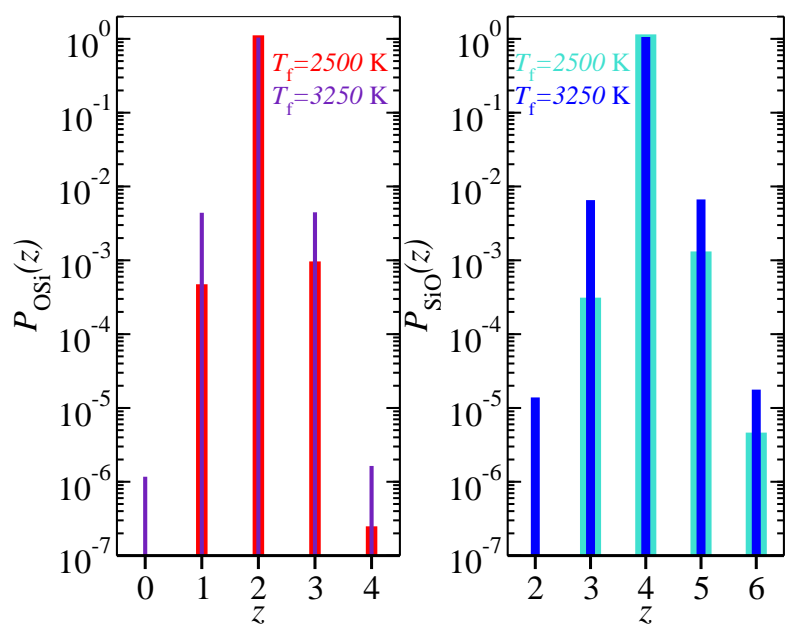

FIG. 6. (color online) Distribution of coordination number $P(z)$ for the number of O-neighbors of an $\mathrm{Si}$-atom $\left(P_{\mathrm{SiO}}\right.$ in left panel) and for the number of $\mathrm{Si}$-neighbors of an $\mathrm{O}$-atom ( $P_{\mathrm{OSi}}$ in right panel). Thick lines are for $T_{\mathrm{f}}=2500 \mathrm{~K}$ and thin dark lines are for $T_{\mathrm{f}}=3250 \mathrm{~K}$.

(thick lines) $99.9 \%$ of Si-atoms are surrounded by four O-atoms and $99.9 \%$ of O-atoms are surrounded by two Si-atoms. Even at $T_{\mathrm{f}}=3250 \mathrm{~K}$ (thin dark lines) there are $98.8 \%$ Si-atoms with $z_{i}^{\mathrm{SiO}}=4$ and $99.1 \%$ O-atoms with $z_{i}^{\mathrm{OSi}}=2$. The time-averaged configurations form an almost perfect O-corner sharing network of $\mathrm{SiO}_{4}{ }^{-}$ tetrahedra. $P_{\mathrm{SiSi}}(z)$ probes this network on the length scale of tetrahedra to tetrahedra connections. Also on this length scale we find that the coordination is almost perfect in the time-averaged configurations: for $T_{\mathrm{f}}=3250 \mathrm{~K}\left(T_{\mathrm{f}}=2500 \mathrm{~K}\right) 95.5 \%(99.1 \%)$ of Si-atoms are surrounded by four Si-atoms. The broadest distribution is $P_{\mathrm{OO}}(z)$ for which $90 \% \mathrm{O}$-atoms are surrounded by $z_{i}^{\mathrm{OO}}=6$ O-atoms.
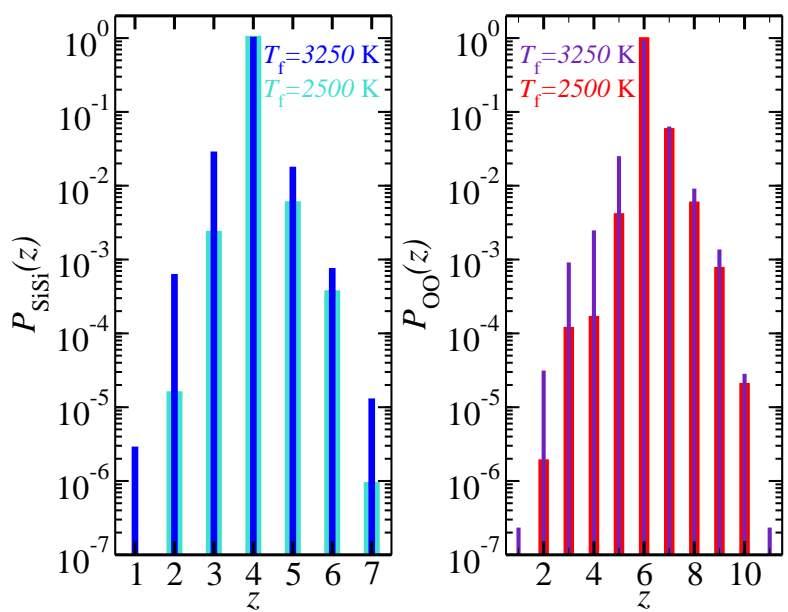

FIG. 7. (color online) Distribution of coordination number $P(z)$ for the number of Si-neighbors of an Si-atom $\left(P_{\mathrm{SiSi}}\right.$ in left panel) and for the number of O-neighbors of an $\mathrm{O}$-atom $\left(\mathrm{P}_{\mathrm{OO}}\right.$ in right panel). Thick lines are for $T_{\mathrm{f}}=2500 \mathrm{~K}$ and thin dark lines are for $T_{\mathrm{f}}=3250 \mathrm{~K}$.

\section{DEFECTS}

In the previous section we showed that the timeaveraged configurations form an almost perfect network with respect to the coordination number $z_{i}^{\alpha \beta}$ with $z_{\text {perfect }}^{\mathrm{SiSi}}=4, z_{\text {perfect }}^{\mathrm{SiO}}=4, z_{\text {perfect }}^{\mathrm{OSi}}=2$ and $z_{\text {perfect }}^{\mathrm{OO}}=6$. For the rest of the paper we observe the dynamics of the system by focusing not on this perfect structure, but rather on the deviations from it.

\section{A. Number of Defects}

We identify defects in the time-averaged structure with help of an indicator function, defined for particle $i$ of type $\alpha$ :

$$
\chi_{i}^{\mathrm{D}}\left(t_{l}, \beta\right)=\left\{\begin{array}{lll}
1 & \text { if at time } t_{l} & z_{i}^{\alpha \beta} \neq z_{\text {perfect }}^{\alpha \beta} \\
0 \text { if at time } t_{l} & z_{i}^{\alpha \beta}=z_{\text {perfect }}^{\alpha \beta}
\end{array} .\right.
$$

This means that an $\alpha \beta$-defect occurs if a particle of type $\alpha$ is surrounded by $z_{i}^{\alpha \beta} \neq z_{\text {perfect }}^{\alpha \beta}$ particles of type $\beta$. In Fig. 8 we show the fraction of particles which are defects,

$$
M_{\alpha \beta}^{\mathrm{D}}=\left\langle\frac{1}{N_{\alpha}} \sum_{i=1}^{N_{\alpha}} \chi_{i}^{\mathrm{D}}\left(t_{l}, \beta\right)\right\rangle .
$$

Consistent with the above coordination number distributions, $M_{\mathrm{SiO}}^{\mathrm{D}}$ and $M_{\mathrm{OSi}}^{\mathrm{D}}$ are very small. Most defects are OO-defects. These findings give further support to the picture of very stable tetrahedra with relaxation processes mainly due to rearrangements of the $\mathrm{SiO}_{4}{ }^{-}$ tetrahedra with respect to each other. With increasing 


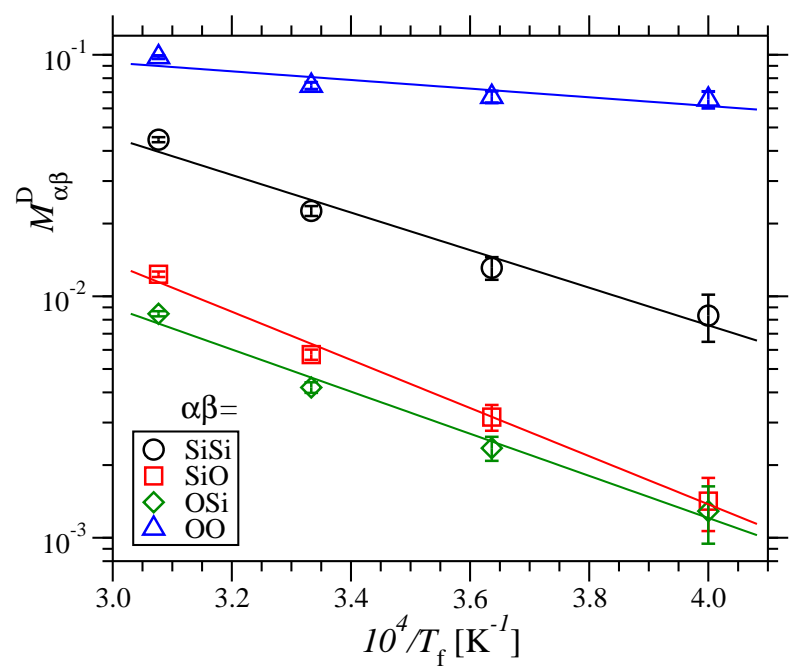

FIG. 8. (color online) $M_{\alpha \beta}^{\mathrm{D}}$, the fraction of particles $i$ of type $\alpha \in\{\mathrm{Si}, \mathrm{O}\}$ which are defects, i.e. for which $z^{\alpha \beta} \neq$ $z_{\text {perfect }}^{\alpha \beta}$ (symbols) versus $1 / T_{\mathrm{f}}$; also shown are Arrhenius fits $M_{\alpha \beta}^{\mathrm{D}}\left(T_{\mathrm{f}}\right)=C \exp \left(\frac{-E_{\mathrm{A}}}{k T_{\mathrm{f}}}\right)$ (lines), with $C=9.8 / 13.4 / 3.7 / 0.3$ and $E_{\mathrm{A}}=1.5 \mathrm{eV} / 2.0 \mathrm{eV} / 1.7 \mathrm{eV} / 0.36 \mathrm{eV}$ for SiSi/SiO/OSi/OOdefects respectively.

temperature, the fraction of defects increases approximately following Arrhenius behavior (with the exception of $M_{\mathrm{OO}}^{\mathrm{D}}$, which is equally well fitted by a power law). These results are in accordance with the work of Horbach and Kob 6] who, however, use non-time-averaged configurations and therefore find more defects.

\section{B. Life Time of Defects via Time Correlation}

So far, we have simply counted the number of defects. Next we look in more detail by observing the defects as they change with time. We ask the question whether the few defects are long lived defects of the same few particles or if instead the defects are short lived, i.e. come and go over the simulation run at different locations. To address this question we define a correlation function for defects of the same particle $i$ occurring at different times $t_{l}$ and $\left(t_{l}+t\right)$ :

$$
\begin{gathered}
C^{\mathrm{DD}}(t, \alpha, \beta)=\left\langle\frac{1}{N_{\alpha}} \sum_{i=1}^{N_{\alpha}} \chi_{i}^{\mathrm{D}}\left(t_{l}, \beta\right) \chi_{i}^{\mathrm{D}}\left(t_{l}+t, \beta\right)\right\rangle \\
-\left\langle\frac{1}{N_{\alpha}} \sum_{i=1}^{N_{\alpha}} \chi_{i}^{\mathrm{D}}\left(t_{l}, \beta\right)\right\rangle\left\langle\frac{1}{N_{\alpha}} \sum_{i=1}^{N_{\alpha}} \chi_{i}^{\mathrm{D}}\left(t_{l}+t, \beta\right)\right\rangle .
\end{gathered}
$$

For the comparison of different $\alpha, \beta$ we normalize by the initial value,

$$
\tilde{C}_{\alpha, \beta}^{\mathrm{DD}}(t)=\frac{C^{\mathrm{DD}}(t, \alpha, \beta)}{C^{\mathrm{DD}}(t=0, \alpha, \beta)}
$$

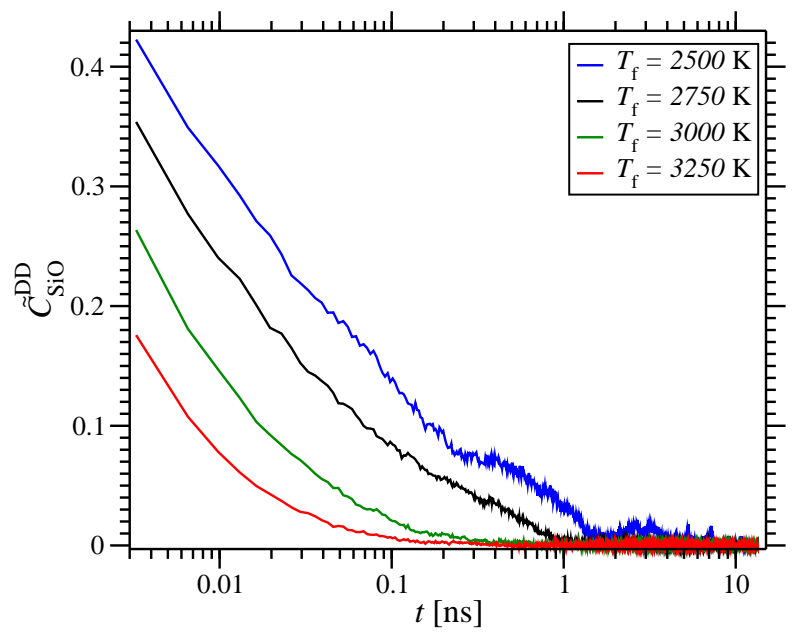

FIG. 9. (color online) Normalized time correlation $\tilde{C}_{\mathrm{SiO}}^{\mathrm{DD}}(t)$ as defined in Eqs. (5) \& (6) for final temperatures $T_{\mathrm{f}}=2500 \mathrm{~K}$ (top curve) to $T_{\mathrm{f}}=3250 \mathrm{~K}$ bottom curve.

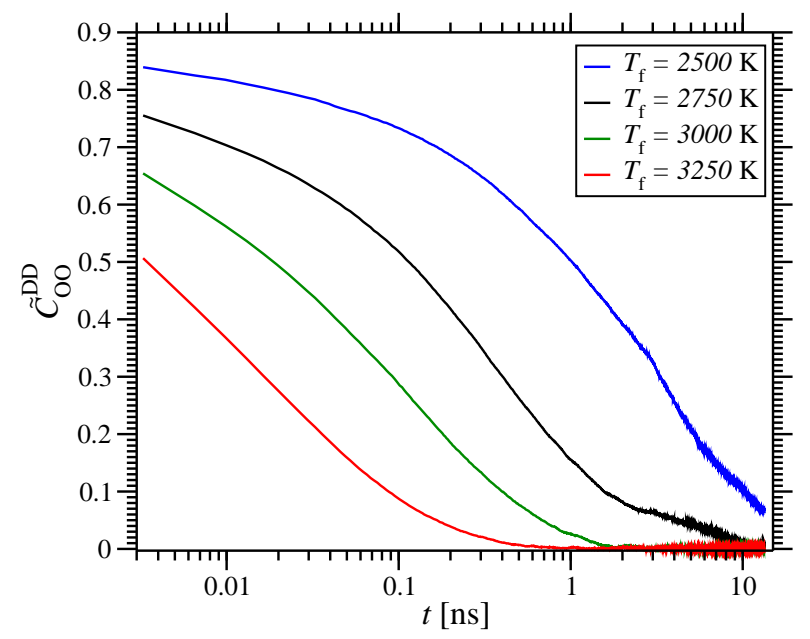

FIG. 10. (color online) Similar to Fig. 9 but here for $\tilde{C}_{\mathrm{OO}}^{\mathrm{DD}}$.

Figs. 9] and 10 reveal a strong temperature dependence: with increasing temperature, all $\tilde{C}_{\alpha \beta}^{\mathrm{DD}}$ decay faster. To quantify this decay for various defect types $\alpha \beta$, we define the lifetime $\tau_{\alpha \beta}^{\mathrm{DD}}$ as the time when $\tilde{C}_{\alpha, \beta}^{\mathrm{DD}}\left(\tau_{\alpha \beta}^{\mathrm{DD}}\right)=0.1$ (we find qualitatively the same results for other values than 0.1). In Fig. 11 we show $\tau_{\alpha \beta}^{\mathrm{DD}}$ as a function of inverse temperature. For all defect types $\alpha, \beta$ the defect lifetime $\tau_{\alpha \beta}^{\mathrm{DD}}\left(T_{\mathrm{f}}\right)$ increases with decreasing $T_{\mathrm{f}}$. The increase is strongest for OO (a factor of 100), and weakest for $\mathrm{SiO}$ (a factor of 20).

For $T_{\mathrm{f}}<T_{\mathrm{c}}$ Horbach and Kob [6] and Saksaengwijit and Heuer 18] find Arrhenius behavior for the life time of SiO-bonds. We therefore compare our data in Fig. 11 with Arrhenius fits (lines). We find good agreement with excitation energies of the same order as found in [18], although refs. 6, 18] discuss the lifetime of bonds instead of defects, as is done here.

One of the most startling observations in Figs. 910 
and 11 is the difference in lifetime for the various types of defects. To judge whether these life times are short or long, we compare with the results of [32] and 31. Single particle (sudden) jump events are of the duration of $\left\langle\Delta t_{\mathrm{d}}\right\rangle=0.01 \mathrm{~ns}$ (see upper arrow in Fig. 11) and the average time spent between successive jumps $\left\langle\Delta t_{\mathrm{b}}\right\rangle$ is shown with filled small symbols. This jump dynamics as well as the incoherent intermediate scattering function become waiting time independent, i.e. the system reaches equilibrium, at $t_{\mathrm{eq}}^{\mathrm{j}} \approx t_{\mathrm{eq}}^{C}$ (see stars in Fig. 11).

We conclude that $\mathrm{SiO}$ and OSi defects survive the duration of a jump but are rather short lived when compared to the time span between jumps; for all $T_{\mathrm{f}}$ the lifetime is less than $0.1 \times\left\langle\Delta t_{\mathrm{b}}\right\rangle$ and for $T_{\mathrm{f}}=3250 \mathrm{~K}$ the lifetime even becomes comparable to the duration of jump events. OO-defects, on the other hand, are of the order of $t_{\mathrm{eq}}^{\mathrm{j}}$, implying that they are the excitations with the longest lifetime in the system under study. Note that all defect lifetimes are substantially longer than $\Delta t_{\mathrm{av}}$, which is indicated by the lower arrow in Fig 11 .

We have checked that these results are robust with respect to choice of $T_{\mathrm{i}}$ and waiting time $t_{\mathrm{w}}$. For $T_{\mathrm{f}}=$ $2500 \mathrm{~K}$ and $2750 \mathrm{~K}$ the results are qualitatively the same for $T_{\mathrm{i}}=3760 \mathrm{~K}$ and $5000 \mathrm{~K}$ and $0 \leq t_{\mathrm{w}} \leq 26.2 \mathrm{~ns}$; for larger temperatures the results agree even quantitatively.

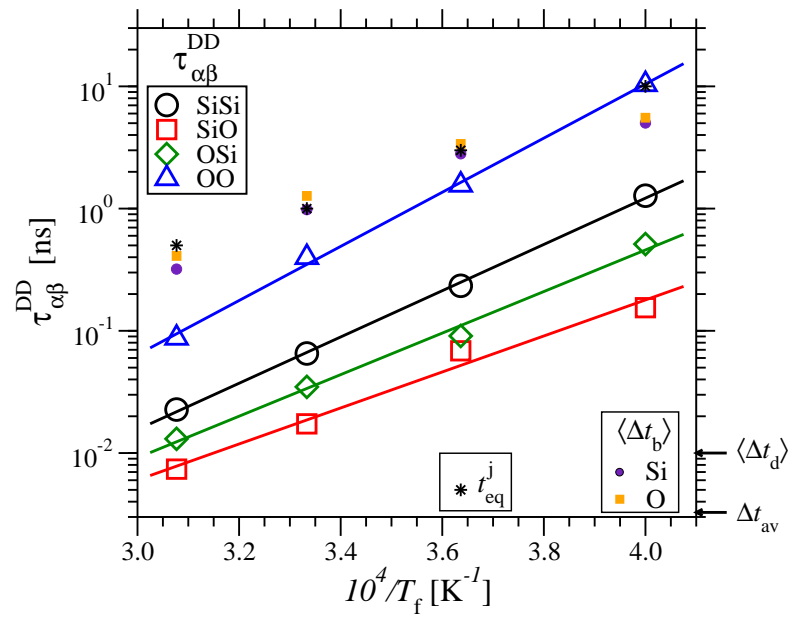

FIG. 11. (color online) $\tau_{\alpha \beta}^{\mathrm{DD}}\left(10^{4} / T_{\mathrm{f}}\right)$ for various $\alpha, \beta \in\{\mathrm{Si}, \mathrm{O}\}$ and for fixed $T_{\mathrm{i}}=5000 \mathrm{~K}$ and $t_{\mathrm{w}}=16.35$ ns (large open symbols). Included are fits (lines) $\tau_{\alpha \beta}^{\mathrm{DD}}=C \exp \left(\frac{E_{\mathrm{A}}}{k T_{\mathrm{f}}}\right)$ with $E_{\mathrm{A}}=3.76 / 2.92 / 3.37 / 4.39 \mathrm{eV}$ for $\mathrm{SiSi} / \mathrm{SiO} / \mathrm{OSi} / \mathrm{OO}$ respectively. For comparison we show the time averaging interval $\Delta t_{\mathrm{av}}$ (lower arrow), the time duration of jumps $\left\langle\Delta t_{\mathrm{d}}\right\rangle$ (upper arrow), the time between successive jumps $\left\langle\Delta t_{\mathrm{b}}\right\rangle$ (small filled symbols), and the equilibrium time $t_{\mathrm{eq}}^{j}$ (stars).

\section{CORRELATION OF JUMPS AND DEFECTS}

Before discussing the correlations of jump events and defects, we briefly recall the methods [32] to identify and analyze jump events.

\section{A. Jumps}

Whereas in the previous section we characterized the relaxation dynamics with defects and their occurrence as function of time $\chi_{i}^{\mathrm{D}}\left(t_{l}, \beta\right)$, we now follow the approach of 32]. For each time-averaged single-particle trajectory $\overline{\mathbf{r}}_{i}\left(t_{l}\right)$ we identify single-particle jump events using Eq. (2) of [32]. This means that a jump event of particle $i$ occurs if

$$
\left|\overline{\mathbf{r}}_{i}\left(t_{l}\right)-\overline{\mathbf{r}}_{i}\left(t_{l-4}\right)\right|>3 \sigma_{\alpha}
$$

holds, where $\sigma_{\alpha}$ is the average fluctuation size for particle $i$ of type $\alpha$. Please note that all following results are qualitatively the same if we use instead of the factor 3 the factor $\sqrt{2}$. Numbering the jump events of particle $i$ by $k$, we determine for each jump event the time $t_{k}^{\text {init }}$ when the particle starts to jump and the time $t_{k}^{\mathrm{f}}$ when the particle jump is finished. We then define an indicator function for the jumpers, in close analogy to the defects, by

$$
\chi_{i}^{\mathrm{J}}\left(t_{l}\right)=\left\{\begin{array}{l}
1 \text { if } t_{k}^{\mathrm{init}} \leq t_{l} \leq t_{k}^{\mathrm{f}} \quad \text { for jumps } k \\
0 \text { otherwise. }
\end{array}\right.
$$

An example for the trajectory in the left panel of Fig. 1 is shown in Fig. 12, clearly revealing the jump events.

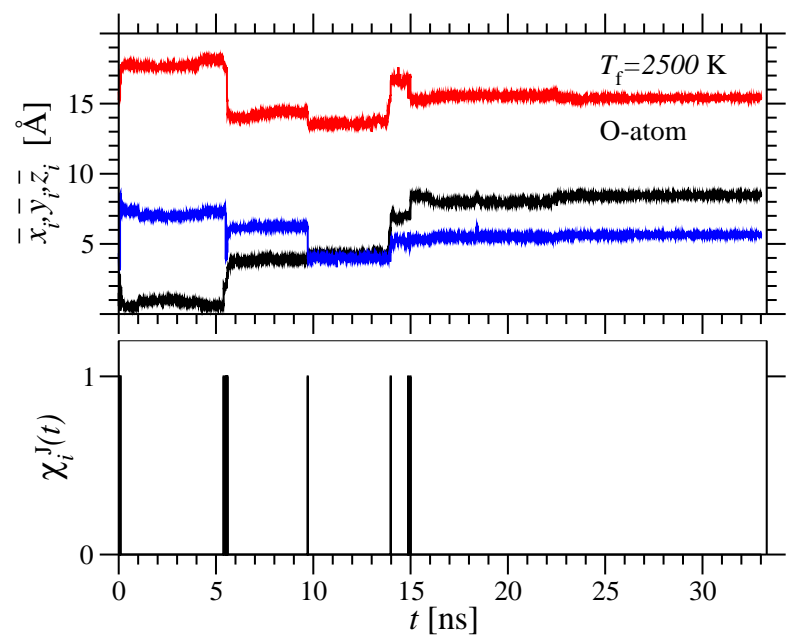

FIG. 12. (color online) Time averaged trajectory of O-atom at $T_{\mathrm{i}}=5000 \mathrm{~K}$ (same as left panel of Fig. 1) and $\chi_{i}^{\mathrm{J}}(t)$ which indicates jump events as horizontal lines.

The average fraction of jumping particles,

$$
M_{\alpha}^{\mathrm{J}}=\left\langle\frac{1}{N_{\alpha}} \sum_{i=1}^{N_{\alpha}} \chi_{i}^{\mathrm{J}}\left(t_{l}\right)\right\rangle,
$$

is shown in Fig. 13. As expected, $M_{\alpha}^{\mathrm{J}}\left(T_{\mathrm{f}}\right)$ is increasing with increasing $T_{\mathrm{f}}$ and can be fitted by MaxwellBoltzmann statistics (lines) with $E_{\mathrm{A}}^{\mathrm{Si}}=E_{\mathrm{A}}^{\mathrm{O}}=2.89 \mathrm{eV}$. Activation energies determined from the diffusion coefficient [6] are considerably higher: $E_{\mathrm{A}}^{\mathrm{Si}}=5.18 \mathrm{eV}$ and $E_{\mathrm{A}}^{\mathrm{O}}=4.66 \mathrm{eV}$. 


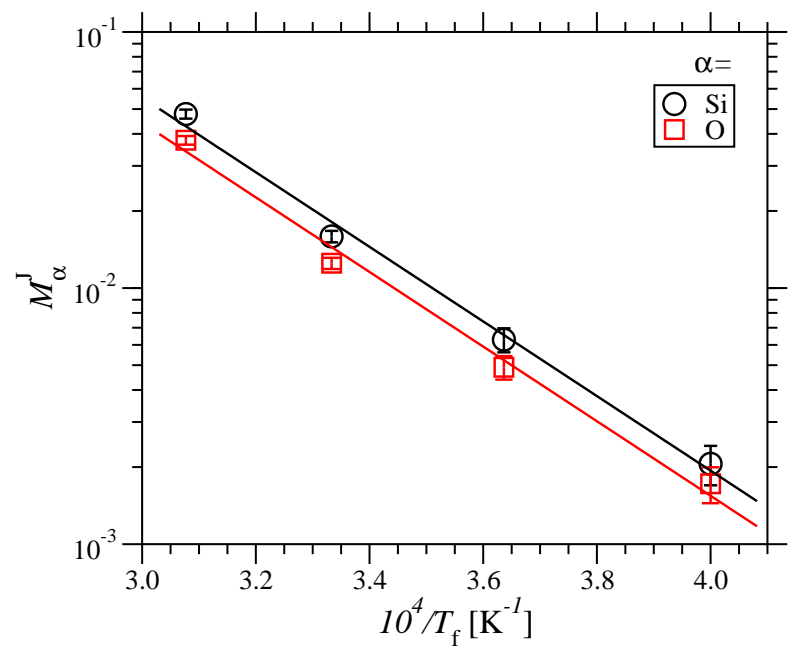

FIG. 13. (color online) The fraction of jumping particles $M_{\alpha}^{\mathrm{J}}$ (symbols) versus $1 / T_{\mathrm{f}}$ and fits with $M_{\alpha}^{\mathrm{J}}\left(T_{\mathrm{f}}\right)=C \exp \left(\frac{-E_{\mathrm{A}}}{k T_{\mathrm{f}}}\right)$ (lines) with $E_{\mathrm{A}}=2.89 \mathrm{eV}$.

\section{B. Correlation of jumps and defects}

So far we have investigated the dynamics of the system from two perspectives: defects and jumps. Having identified jump events by $\chi_{i}^{\mathrm{J}}\left(t_{l}\right)$ and defects by $\chi_{i}^{\mathrm{D}}\left(t_{l}, \beta\right)$, we can now quantify the correlations between the two sorts of events. To illustrate our approach, we show in Fig. 14 $\chi_{i}^{\mathrm{J}}\left(t_{l}\right)$ and $\chi_{i}^{\mathrm{D}}\left(t_{l}, \beta\right)$ for an $\mathrm{O}$ jumper whose trajectory is shown in Fig. 1. A similar plot for an Si jumper is shown in Fig. 14. If the horizontal lines of $\chi_{i}^{\mathrm{D}}(t, \beta)$ and $\chi_{i}^{\mathrm{J}}(t)$ are aligned, this implies a strong correlation of jumpers and defects.

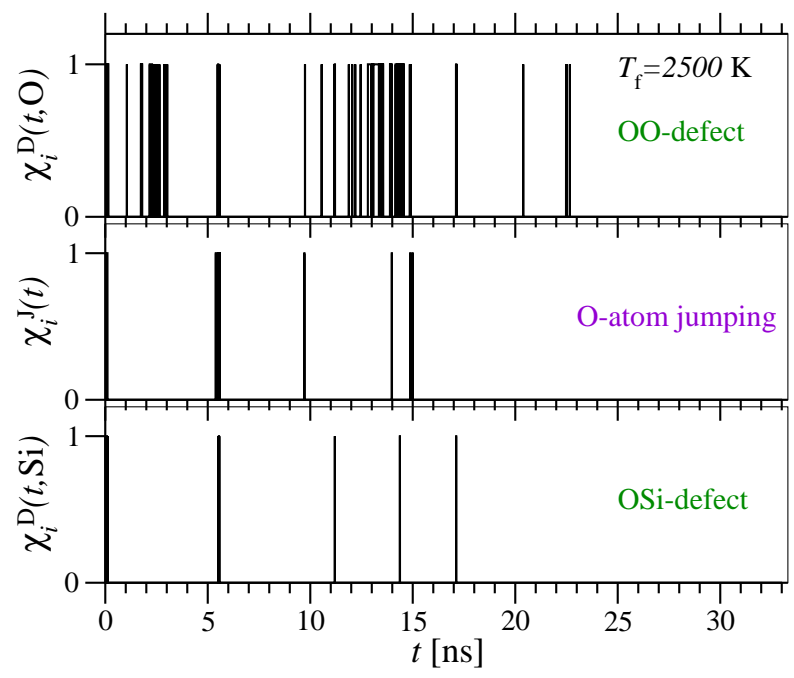

FIG. 14. For the comparison we show for an O-atom the defect functions $\chi_{i}^{\mathrm{D}}(t)$ of OO-defects (top figure) and of OSi-defects (bottom figure) and the jump function $\chi_{i}^{\mathrm{J}}(t)$ (middle figure) at $T_{\mathrm{f}}=2500 \mathrm{~K}$.

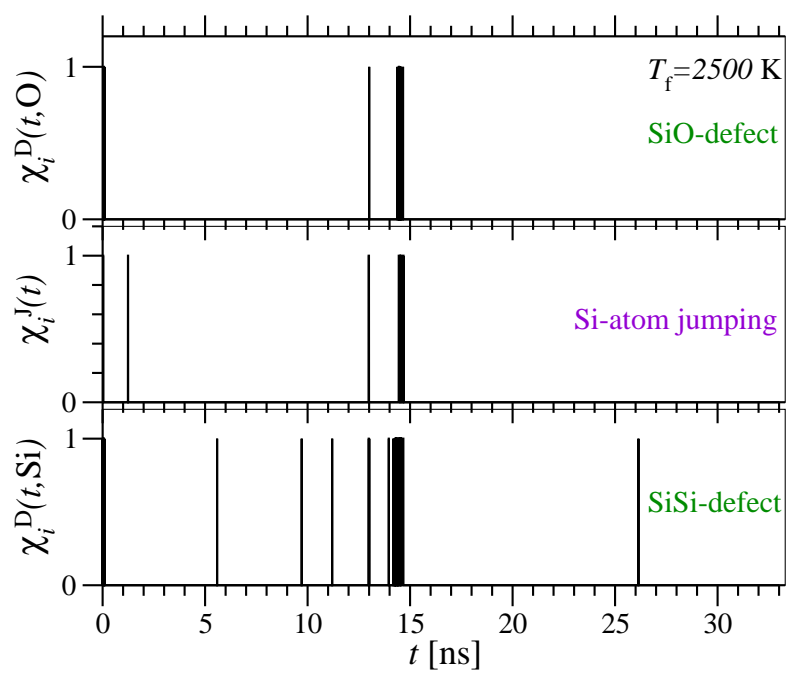

FIG. 15. Similar to Fig. 14 also at $T_{\mathrm{f}}=2500 \mathrm{~K}$ but for an Si-atom we show the defect functions $\chi_{i}^{\mathrm{D}}(t)$ of SiO-defects (top figure) and of SiSi-defects (bottom figure) and the jump function $\chi_{i}^{\mathrm{J}}(t)$ (middle figure).

Both jumping atoms as well as defects are rare events at low temperatures. If atoms were jumping independently from creating defects then the joint probability that an atom is a jumper and simultaneously a defect would be given by the product of two very small probabilities, which can be estimated as follows. The probability $p$ for a particle to be a jumper can be approximated by the fraction of jumpers $p=M_{\alpha}^{J}$ and similarly the probability $q$ for a particle to be a defect by $q=M_{\alpha, \beta}^{D}$. If the events were independent, the joint probability for a particle to be a defect and simultaneously a jumper is $p q$. At $T_{f}=2500 \mathrm{~K}$ the smallest value of $p q$ is $2 \times 10^{-6}$ for OSi defects and the largest value of $p q$ is $10^{-4}$ for OO defects. In contrast Figs. 14 and 15 indicate a larger likelihood of $\chi_{i}^{\mathrm{D}}$ and $\chi_{i}^{\mathrm{J}}$ being aligned and thus suggest a strong correlation.

A quantitative measure for the correlations of jumpers and defects is the following correlation function: 


$$
A_{\alpha, \beta}^{\mathrm{DJ}}=\frac{\left\langle\frac{1}{N_{\alpha}} \sum_{i=1}^{N_{\alpha}} \chi_{i}^{\mathrm{D}}\left(t_{l}, \beta\right) \chi_{i}^{\mathrm{J}}\left(t_{l}\right)\right\rangle-\left\langle\frac{1}{N_{\alpha}} \sum_{i=1}^{N_{\alpha}} \chi_{i}^{\mathrm{D}}\left(t_{l}, \beta\right)\right\rangle\left\langle\frac{1}{N_{\alpha}} \sum_{i=1}^{N_{\alpha}} \chi_{i}^{\mathrm{J}}\left(t_{l}\right)\right\rangle}{\left\langle\frac{1}{N_{\alpha}} \sum_{i=1}^{N_{\alpha}} \chi_{i}^{\mathrm{D}}\left(t_{l}, \beta\right)\right\rangle\left\langle\frac{1}{N_{\alpha}} \sum_{i=1}^{N_{\alpha}} \chi_{i}^{\mathrm{J}}\left(t_{l}\right)\right\rangle} .
$$

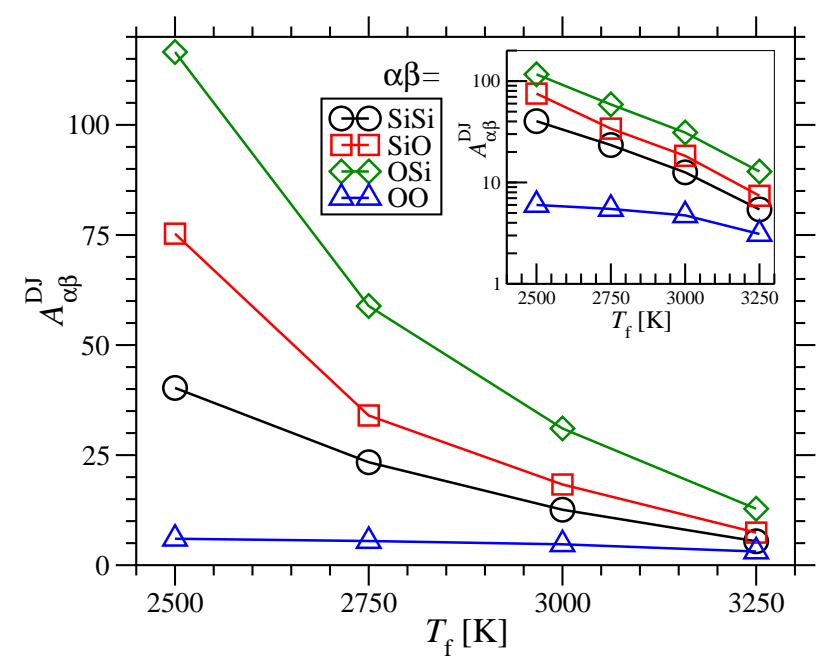

FIG. 16. Correlation of defects and jumps $A_{\alpha \beta}^{\mathrm{DJ}}$ as defined in Eq. (10). The lines are a guide to the eye. The inset shows the same data in a semilogarithmic plot.

The defect-jumper correlation is shown in Fig. 16 for both types of jumpers (indicated by the first letters) and both types of corresponding defects (indicated by the last letters). The correlation of a defect involving a wrong coordination between an Si-atom and an O-atom is very high at low temperatures for both $\mathrm{Si}$-atoms and O-atoms jumping (red and green line respectively). Only the correlation between an O-atom which is jumping and not correctly coordinated with other O-atoms is less well pronounced. We interpret these results as follows: A breakup of the $\mathrm{SiO}_{4}$ tetrahedra, which destroys the appropriate coordination between $\mathrm{Si}$ and $\mathrm{O}$-atoms, is likely to involve a jump, whereas jumps of an O-atom, involving the motion of two tetrahedra with respect to each other, can happen without the creation of a defect.

At high temperatures the correlation of jumpers and defects is small, which can also be guessed from single trajectories, see Fig. 17. It is also apparent from the single trajectory (Fig. 17) that the O-atom which jumps is very often simultaneously an OSi defect, whereas OO defects are so frequent that hardly any correlation can be detected. This is reflected in the average correlation (see Fig. 16) of the O-jumper which is four times higher for OSi defects than for OO-defects at $T=3250 \mathrm{~K}$.

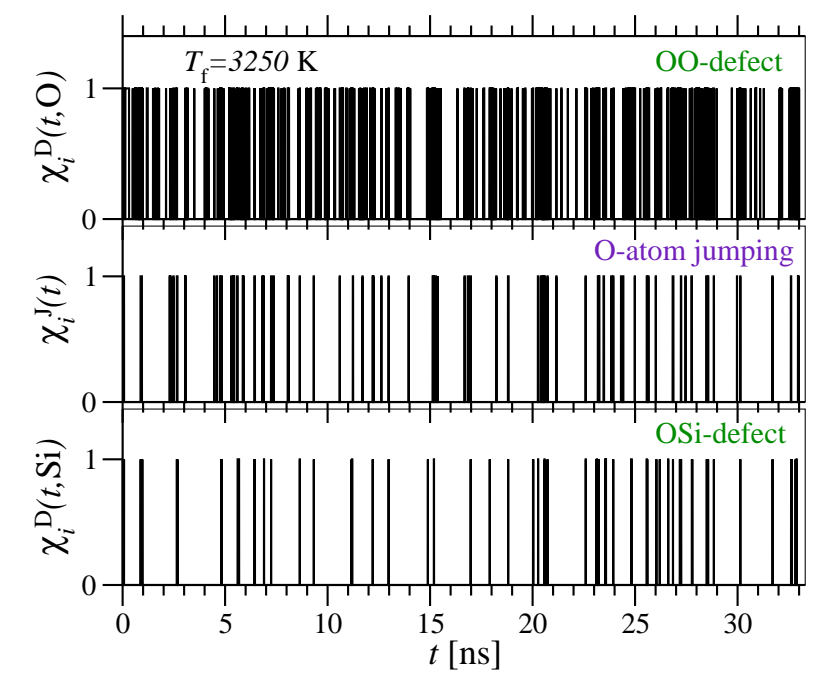

FIG. 17. Similar to Fig. 14 for the same O-atom but here for $T_{\mathrm{f}}=3250 \mathrm{~K}$ we show the defect functions $\chi_{i}^{\mathrm{D}}(t)$ of OO-defects (top figure) and of OSi-defects (bottom figure) and the jump function $\chi_{i}^{J}(t)$ (middle figure).

\section{SUMMARY AND CONCLUSIONS}

In this paper we analyzed time-averaged single particle trajectories $\overline{\mathbf{r}}_{i}\left(t_{l}\right)$ at temperatures well below the glass transition temperature. Loosely spoken, the time average allowed us to watch a movie of the complicated particle dynamics by filtering out the background noise of vibrations, revealing the underlying major relaxational processes. Using $\overline{\mathbf{r}}_{i}\left(t_{l}\right)$ we determined the radial distribution function and coordination number distribution. Both are very sharply peaked, reflecting a highly structured network of O-corner sharing $\mathrm{SiO}_{4}$-tetrahedra in which almost all particles have the ideal coordination number. This led us to focus on deviations from this well-defined local neighborhood in order to find the excitations which are responsible for the slow structural relaxation in the random network.

We defined defects in the time-averaged structure by an indicator function: $\chi_{i}^{D}\left(t_{l}, \beta\right)=1$, if particle $i$ of type $\alpha$ at time $t_{l}$ has coordination $z_{i}^{\alpha \beta}\left(t_{l}\right) \neq z_{\text {perfect }}^{\alpha \beta}$. We computed the average number of defects and the time-delayed autocorrelation of $\chi_{i}^{D}\left(t_{l}, \beta\right)$, from which we extracted the average lifetimes of defects. We observe a very strong variation of lifetimes for different sorts of defects. SiOand OSi-defect correlations decay fast; in the movie analogy they correspond to short flashes which come and go. (Note, however, that we are looking at time scales which 
are larger than jump times and huge as compared to oscillation periods.) In contrast, OO-defects are very long lived; their lifetime becomes comparable to the equilibration time for $T=2750 \mathrm{~K}$. All lifetimes are strongly temperature dependent. E.g. the lifetime of OO-defects increases by a factor of 100 in the temperature range $2500 \mathrm{~K} \leq T \leq 3250 \mathrm{~K}$. Given the rather mild temperature dependence of the average structure as described by the pair correlation, we expect that defects are one of possibly other excitations which determine the temperature dependence of glassy properties at low temperatures. This issue needs to be explored further in future work.

Local structural rearrangements are achieved by jumping atoms, i.e. atoms which move considerably further than a typical oscillation amplitude. The statistics of jumpers in $\mathrm{SiO}_{2}$ has been studied in previous work [31, 32]. We expect that single particle jump events go hand in hand with the creation and annihilation of defects and hence we have computed the correlation of defect and jump events. At low temperatures the correlation is very strong, in particular for defects involving one Si- and one O-atom, emphasizing the important role of defects for structural rearrangements.

It would be interesting to investigate spatial correlations of defect and jump events and relate our work to studies of dynamic heterogeneities in which the most mobile particles are selected (for reviews we refer the reader to [2, 39]). Spatial correlations, however, require a larger simulation size, which is planned for the future. Another interesting extension are other network formers such as $\mathrm{B}_{2} \mathrm{O}_{3}$ and $\mathrm{BeF}_{2}$, for which we expect defects to be well defined. We leave it for future work to study their defect dynamics as it is presented here. Our analysis of time-averaged trajectories shines light on the main features of structural changes and is easily applicable to simulations and experiments of other network-forming and non-network forming systems and other strong and fragile glass formers. It remains to be seen whether the defect and jump dynamics presented here is a universal phenomenon.

\section{ACKNOWLEDGMENTS}

KVL was supported by the Deutsche Forschungsgemeinschaft via SFB 602 and FOR1394. KVL thanks the Institute of Theoretical Physics, University of Göttingen, for financial support and hospitality. We thank B. Vollmayr-Lee for fruitful discussions and comments on an earlier version of this manuscript.
[1] K. Binder and W. Kob, Glassy Materials and Disordered Solids - An Introduction to Their Statistical Mechanics (World Scientific, Singapore, 2005).

[2] M. Ediger, Annu. Rev. Phys. Chem. 51, 99 (2000).

[3] C. A. Angell, Science 267, 1924 (1995).

[4] C. A. Angell and W. Sichina, Ann. New York Acad. Sci. 279, 53 (1976).

[5] C. A. Angell, Non-Cryst. Solids 131, 13 (1991).

[6] J. Horbach and W. Kob, Phys. Rev. B 60, 3169 (1999).

[7] C. A. Angell and M. Hemmati, AIP Conf. Proc. 1518, 9 (2013).

[8] B. W. H. van Beest, G. J. Kramer, and R. A. van Santen, Phys. Rev. Lett. 64, 1955 (1990).

[9] K. Vollmayr, W. Kob, and K. Binder, Phys. Rev. B 54, 15808 (1996).

[10] J. Badro, D. M. Teter, R. T. Downs, P. Gillet, R. J. Hemley, and J.-L. Barrat, Phys. Rev. B 56, 5797 (1997).

[11] S. N. Taraskin and S. R. Elliott, Phys. Rev. B 59, 8572 (1999).

[12] I. Saika-Voivod, F. Sciortino, T. Grande, and P. H. Poole, Phys. Rev. E 70, 061507 (2004).

[13] I. Saika-Voivod, P. H. Poole, and F. Sciortino, Nature 412, 514 (2001).

[14] J. Badro, P. Gillet, and J.-L. Barrat, Europhys. Lett. 42, 643 (1998).

[15] J.-L. Barrat, J. Badro, and P. Gillet, Mol. Simul. 20, 17 (1997).

[16] A. Saksaengwijit, J. Reinisch, and A. Heuer, Phys. Rev. Lett. 93, 235701 (2004).

[17] J. Reinisch and A. Heuer, Phys. Rev. Lett. 95, 155502 (2005).

[18] A. Saksaengwijit and A. Heuer, Phys. Rev. E 73, 061503
(2006).

[19] A. Saksaengwijit and A. Heuer, J. Phys.: Condens. Matter 19, 205143 (2007).

[20] J. Reinisch and A. Heuer, J. Phys. Chem. B 110, 19044 (2006).

[21] P. Scheidler, W. Kob, A. Latz, J. Horbach, and K. Binder, Phys. Rev. B 63, 104204 (2001).

[22] S. N. Taraskin and S. R. Elliott, Phys. Rev. B 56, 8605 (1997).

[23] S. N. Taraskin and S. R. Elliott, Physica B 316, 81 (2002).

[24] T. Uchino, J. D. Harrop, S. N. Taraskin, and S. R. Elliott, Phys. Rev. B 71, 014202 (2005).

[25] F. Leonforte, J. Non-Cryst. Solids 357, 552 (2011).

[26] M. Vogel and S. C. Glotzer, Phys. Rev. E 70, 061504 (2004).

[27] M. N. J. Bergroth, M. Vogel, and S. C. Glotzer, J. Phys. Chem. B 109, 6748 (2005).

[28] V. Teboul, Eur. Phys. J. B 51, 111 (2006).

[29] P. K. Hung, N. T. T. Ha, and N. V. Hong, Eur. Phys. J. E 36, 60 (2013).

[30] L. Berthier, Phys. Rev. Lett. 98, 220601 (2007).

[31] K. Vollmayr-Lee, J. A. Roman, and J. Horbach, Phys. Rev. E 81, 061203 (2010).

[32] K. Vollmayr-Lee, R. Bjorkquist, and L. M. Chambers, Phys. Rev. Lett. 110, 017801 (2013).

[33] This is not a complete list of BKS-simulations. For further work please see references therein.

[34] F. H. Stillinger and T. A. Weber, J. Chem. Phys. 80, 4434 (1984).

[35] S. Sastry, P. G. Debenedetti, F. H. Stillinger, T. B. Schrøder, J. C. Dyre, and S. C. Glotzer, Physica A 270, 
301 (1999).

[36] C. Oligschleger and H. R. Schober, Phys. Rev. B 59, 811 (1999).

[37] M. Kluge and H. R. Schober, Phys. Rev. B 70, 224209
(2004).

[38] A. S. Keys, L. O. Hedges, J. P. Garrahan, S. C. Glotzer, and D. Chandler, Phys. Rev. X 1, 021013 (2011).

[39] G. Biroli and J. P. Garrahan, J. Chem. Phys. 138, 12A301 (2013). 